\title{
Hypermethylation of WIF1 and its inhibitory role in the tumor growth of endometrial adenocarcinoma
}

\author{
XINCHAO DENG, CONGZHE HOU, HUALI WANG, TINGTING LIANG and LIN ZHU
}

Department of Obstetrics and Gynecology, The Second Hospital of Shandong University, Jinan, Shandong 250012, P.R. China

Received May 6, 2017; Accepted July 12, 2017

DOI: $10.3892 / \mathrm{mmr} .2017 .7564$

\begin{abstract}
Endometrial carcinoma is the most common malignancy of the female genital tract and is the fourth most common malignancy among women worldwide. Endometrial adenocarcinoma (EAC) accounts for $\sim 80 \%$ of endometrial carcinoma cases. Numerous critical genetic events have been determined to serve an essential role in EAC progression; however, the precise molecular mechanisms underlying EAC progression remain unclear. Pyrosequencing and methylation-specific PCR were used to detect the methylation status of Wnt inhibitory factor 1 (WIF1). Immunohistochemistry and western blot were used to detect the expression of WIF1, Wnt family member 1 and other related pathways. The anticancer role of WIF1 in EAC was investigated in vitro and in vivo. Two of the three EAC cases exhibited significantly high methylation in five $\mathrm{CpG}$ sites, and the WIF1 methylation rate in EAC and endometrial tissues was 43.4 and $8 \%$, respectively $(\mathrm{P}<0.05)$. The kappa consistency coefficient was -0.369 between methylation and mRNA expression $(\mathrm{P}<0.05)$ and WIF1 expression levels were significantly downregulated in EAC tissues compared with non-tumorous tissues $(\mathrm{P}<0.01)$. The 5-year overall survival rates were significantly lower for patients with tumors that negatively expressed WIF1 when compared with the $77.9 \%$ exhibited by those with positive WIF1 expression. Furthermore, the proliferation rate of KLE cells was significantly reduced following 5-aza-20-deoxycytidine treatment or WIF1 overexpression in vitro and in vivo, which may be associated with downregulated c-Myc and phosphorylated-extracellular signal-regulated kinase expression. These results demonstrated the important role of WIF1 in EAC tumorigenesis, and suggested that WIF1 may be a potential drug target in EAC treatment.
\end{abstract}

Correspondence to: Dr Lin Zhu, Department of Obstetrics and Gynecology, The Second Hospital of Shandong University, 247 Beiyuan Da Street, Tianqiao, Jinan, Shandong 250012, P.R. China E-mail: linzhu0205@126.com

Key words: endometrial adenocarcinoma, methylation, proliferation, Wnt inhibitory factor 1, c-Myc, extracellular signal-regulated kinase

\section{Introduction}

Endometrial carcinoma is the most common malignancy of the female genital tract and is the fourth most common malignancy among women worldwide (1). Endometrial adenocarcinoma (EAC) is a type I endometrial carcinoma, which accounts for $\sim 80 \%$ of cases, and is often associated with obesity, estrogen stimulation and unfavorable prognosis (2). Numerous critical genetic events, including microsatellite instability and alterations in the phosphatase and tensin homolog signaling pathway, have been revealed to serve essential roles in EAC progression; however, the precise molecular mechanisms underlying EAC progression remain unclear $(2,3)$.

The Wnt signaling pathway is a major regulator of cell proliferation, migration and differentiation, embryogenesis, adult tissue homeostasis and tumor progression (4). Aberrant activation of the $\mathrm{Wnt} / \beta$-catenin signaling pathway contributes to the progression of numerous major human cancers (5). Wnt inhibitory factor 1 (WIF1) binds directly to extracellular Wnt ligands, preventing receptor interactions and inducing $\beta$-catenin degradation. Downregulation of WIF1 has been detected in human prostate, breast, lung and bladder cancers. Recently, WIF1 silencing caused by promoter hypermethylation was reported in gastrointestinal, lung and bladder cancers (6-8). In addition, Wnt pathway activation has been revealed to be involved in EAC (9); however, to the best our knowledge, the expression and precise role of WIF1 in EAC progression have yet to be determined.

The present study aimed to investigate WIF1 gene promoter methylation, WIF1 mRNA and protein expression, and the association between WIF1 and the prognosis of patients with EAC. An EAC cell line was also treated with a demethylating agent to determine whether demethylation was able to restore WIF1 expression. The role of the WIF1 gene in cell proliferation and tumor growth of the EAC cell line, KLE, and subsequently, its effects on the expression of proteins in the non-canonical Wnt pathway, including c-Myc and extracellular signal-regulated kinase (ERK), were also evaluated.

\section{Materials and methods}

Tissue samples. A total of 106 EAC samples (age, $59.2 \pm 10.9$ years), acquired previously by the Department of Obstetrics and Gynecology, The Second Hospital of Shandong University (Jinan, China) between January 2006 
and June 2008, were provided for use in the present study. In addition, 50 normal endometrial tissue specimens (age, $40.2 \pm 7.3$ years) were obtained from women who underwent hysterectomy or endometrial curettage for non-EAC-associated diseases between January 2006 and June 2008, such as uterine leiomyoma or prolapse. None of the patients received chemotherapy or radiation therapy prior to surgery. The follow-up information for the EAC cases was collected previously and was obtained for the present study from the department's follow-up system. The follow-up time was between 14 and 90 months $(60.2 \pm 19.3$ months). The EAC tissues were of typical histology and were comprised of $>70 \%$ tumor cells. The procedures, and the use of all specimens and clinical information, were approved by the Clinical Research Ethics Committee of the Second Hospital of Shandong University.

Cell culture and in vitro demethylation treatment. The human endometrial cancer cell line KLE was obtained from the American Type Culture Collection (Manassas, VA, USA). KLE cells were cultured in Dulbecco's modified Eagle's medium supplemented with $10 \%$ heat inactivated fetal bovine serum (both from Gibco; Thermo Fisher Scientific, Inc., Waltham, MA, USA) at $37^{\circ} \mathrm{C}$ in a humidified atmosphere containing $5 \%$ $\mathrm{CO}_{2}$; the culture medium was changed every 2-3 days. For demethylation treatment, $1 \times 10^{6} \mathrm{KLE}$ cells were seeded into 6 -well plates and cultured for $24 \mathrm{~h}$ at $37^{\circ} \mathrm{C}$. Subsequently, cells were exposed to $30 \mu \mathrm{mol} / 15$-aza-20-deoxycytidine (5-Aza-dC; Sigma-Aldrich; Merck KGaA, Darmstadt, Germany) and fresh demethylating agent was added every $24 \mathrm{~h}$ for 7 days at $37^{\circ} \mathrm{C}$. Cells that were not treated with $5-\mathrm{Aza}-\mathrm{dC}$ were considered control cells.

$R N A$ extraction and reverse transcription-quantitative polymerase chain reaction $(R T-q P C R)$. Total RNA was isolated using TRIzol reagent (Invitrogen; Thermo Fisher Scientific, Inc.) from EAC and control tissues according to the manufacturer's protocol. cDNA was synthesized using the GoScript reverse transcriptase system and random hexamer primers (Promega Corporation, Madison, WI, USA) according to the manufacturer's protocol. RT-qPCR was performed using a relative quantification protocol by SYBR Fast qPCR Mix (Takara Bio, Inc., Otsu, Japan). GAPDH was used as an internal control. The primer sequences were as follows: WIF1 forward, 5'-CCGAAATGGAGGCTTTTGTA-3' and reverse, 5'-GTG TCTTCCATGCCAACCTT-3'; GAPDH forward, 5'-ACA ACTTTGGTATCGTGGAAGG-3' and reverse, 5'-GCCATC ACGCCACAGTTTC-3'. The thermocycling conditions were as follows: $95^{\circ} \mathrm{C}$ for $5 \mathrm{~min}$ followed by 40 cycles at $95^{\circ} \mathrm{C}$ for $30 \mathrm{sec}, 52^{\circ} \mathrm{C}$ for $30 \mathrm{sec}$ and $72^{\circ} \mathrm{C}$ for $30 \mathrm{sec}$, and a final extension step at $72^{\circ} \mathrm{C}$ for $10 \mathrm{~min}$. The relative fold change in mRNA expression compared with the control was calculated using the comparative Cq method, $2^{-\Delta \Delta C q}(10)$.

Genomic DNA extraction, pyrosequencing and methylationspecific PCR (MSP). Genomic DNA was isolated from KLE cells and patient tissues using the Blood and Cell Culture DNA Mini kit (Qiagen, Inc., Valencia, CA, USA) according to the manufacturer's protocol. Bisulfite genomic DNA modification and purification was performed using EpiTect Bisulfite kit (Qiagen, Inc.) according to the manufacturer's instruction.
Three EAC and two normal tissue samples were selected to undergo pyrosequencing uing PyroMark Q24 reagents (Qiagen GmbH, Hilden, Germany) according to the manufacturer's instructions. The PyroMark MD system and PyroMark $\mathrm{CpG}$ software version 1.0 (Qiagen $\mathrm{GmbH}$ ) were used to measure the methylation frequency of the WIF1 promoter CpG sites (5'-TTTATTTCYCYGGCYTTTTATTGGGCY TATCYTATTG-3'). Methylation was analyzed using MSP and the following MSP primers were used: Methylated WIF1 forward, 5'-CGTTTTATTGGGCGTATCGT-3' and reverse, 5'-ACTAACGCGAACG-AAATACGA-3'; and unmethylated WIF1 forward, 5'-GGGTGTTTTATTGGG-TGTATTGT-3' and reverse, 5'-AAACCAACAATCAACAAAAC-3'. PCR was performed in a $30 \mu 1$ reaction volume using HotStarTaq DNA polymerase (Qiagen $\mathrm{GmbH}$ ). The thermocycling conditions were as follows: $95^{\circ} \mathrm{C}$ for $5 \mathrm{~min}$ followed by 35 cycles at $95^{\circ} \mathrm{C}$ for $30 \mathrm{sec}, 60^{\circ} \mathrm{C}$ for $30 \mathrm{sec}$ and $72^{\circ} \mathrm{C}$ for $30 \mathrm{sec}$, and a final extension at $72^{\circ} \mathrm{C}$ for $5 \mathrm{~min}$. Products were then separated by $2 \%$ agarose gel electrophoresis and visualized by ethidium bromide under ultraviolet illumination. The methylation rate was calculated using the following equation: Methylated/(methylated + unmethylated). Methylation $>20 \%$ was set as the cut off value.

Immunohistochemistry (IHC). Formalin-fixed, paraffinembedded tissue blocks were cut into $4-\mu \mathrm{m}$ sections and mounted onto charged glass slides, deparaffinized and rehydrated in a graded series of ethanol. Antigen retrieval was performed in $1 \mathrm{mmol} / 1$ EDTA solution $\left(\mathrm{pH} \mathrm{8.0)}\right.$ at $98^{\circ} \mathrm{C}$ for $15 \mathrm{~min}$. Endogenous peroxidase activity was blocked with $3.0 \%$ hydrogen peroxide for $5 \mathrm{~min}$ at room temperature. Sections were then blocked in $10 \%$ normal goat serum with $1 \%$ bovine serum albumin (both from Sigma-Aldrich; Merck KGaA) in Tris-buffered saline (TBS) with $0.025 \%$ Tween-20 (TBST) for $1 \mathrm{~h}$ at $37^{\circ} \mathrm{C}$. Subsequently, the sections were incubated with anti-WIF1 rabbit monoclonal antibody (1:250; ab89935; Abcam, Cambridge, MA, USA) in blocking buffer at $4^{\circ} \mathrm{C}$ overnight. Following three washes, the sections were incubated with horseradish peroxidase-conjugated goat anti-rabbit secondary antibody for $1 \mathrm{~h}$ (ab6721, 1:500 dilution; Abcam). Visualization was performed using the DAB peroxidase substrate kit (cat no. SK-4705; Vector Laboratories, Inc., Burlingame, CA, USA) in Milli-Q purified water (EMD Millipore, Billerica, MA, USA). The slides were counterstained with Mayer's hematoxylin at room temperature, and the sections were dehydrated, cleared, mounted and observed by light microscopy. Negative controls were run on all sections by incubating with 3\% normal goat serum in TBST, generated against unassociated antigens. Finally, the results of IHC were semi-quantified; the percentage of positive staining was scored as follows: i) 0 , ii) $1(1-25 \%)$, iii) 2 (26-50\%), iv) $3(51-75 \%)$ and v) 4 (76-100\%). The intensity of positive staining was scored as follows: i) 0 (negative), ii) 1 (weak), iii) 2 (moderate) and iv) 3 (strong). A percentage $>50 \%$ and a moderate intensity score were used as the positive cut-off criteria.

Western blotting. Briefly, endometrial cancer KLE cells were lysed for $30 \mathrm{~min}$ at $4^{\circ} \mathrm{C}$ in lysis buffer (Sigma-Aldrich; Merck KGaA). The bicinchoninic acid method was used to 
determine the protein concentration. Equal amounts of protein extracts $(10 \mu \mathrm{g})$ were separated by $10 \%$ SDS-PAGE and were transferred to polyvinylidene difluoride membranes (Bio-Rad Laboratories, Inc., Hercules, CA, USA), which were blocked with $5 \%$ BSA at room temperature for $1 \mathrm{~h}$. The membranes were then incubated overnight at $4^{\circ} \mathrm{C}$ with: Anti-WIF1 (ab89935, 1:500 dilution), anti-Wnt family member 1 (ab15251, 1:500 dilution) (both from Abcam), anti-ERK/p-ERK (\#4695/\#4370, 1:500 dilution), anti-c-Myc (\#13987, 1:500 dilution) (all from Cell Signaling Technology, Inc., Danvers, MA, USA) or anti- $\beta$-actin (A2066, 1:1000 dilution; Sigma-Aldrich; Merck KGaA) antibodies. Following washing, the membranes were incubated with anti-rabbit or anti-mouse HRP-labeled secondary antibodies (\#7074 or \#7076, 1:2,000 dilution; Cell Signaling Technology, Inc.) at room temperature for $2 \mathrm{~h}$. Finally, the blots were developed using an enhanced chemiluminescence detection system (Amersham Life Science; GE Healthcare, Chicago, IL, USA). The expression of $\beta$-actin served as the loading control. Densitometric analyses were performed using Bio-Rad Quantity One software version 4.3.0 (Bio-Rad Laboratories, Inc.). The intensity of the bands of each treatment was compared with the intensity of the control.

Plasmid construction and cell transfection. The full-length WIF1 cDNA was PCR cloned, sequence-verified and further subcloned into the retroviral expression vector pBABE-puro (Addgene, Inc., Cambridge, MA, USA) to generate pBABE-puro-WIF1. For stable transfection, KLE cells were seeded into 24-well culture plates at a density of $3 \times 10^{4}$ cells/well and transfected with pBABE-puro-WIF1 or pBABE-puro empty vector using Lipofectamine 2000 (Invitrogen; Thermo Fisher Scientific, Inc.) according to the manufacturer's protocol. Transfection was performed at a DNA concentration of $800 \mathrm{ng} /$ well. Following transfection, stable clones were selected using puromycin $(2 \mu \mathrm{g} / \mathrm{ml})$ starting at $48 \mathrm{~h}$ post-transfection, and the expression of the transgene was detected by western blotting and RT-qPCR.

Proliferation assay. To determine the effects of WIF1 on the proliferation of KLE cells, cells $\left(4 \times 10^{3} /\right.$ well) transfected as aforementioned with pBABE-WIF1 and empty vector were seeded in 96-well culture plates in complete medium in triplicate and incubated at $37^{\circ} \mathrm{C}$ with $5 \% \mathrm{CO}_{2}$ in a humidified incubator for 1-6 days. Cell proliferation assay was performed using Cell Counting Kit-8 (CCK-8; Dojindo Molecular Technologies, Inc., Rockville, MD, USA) every day according to the manufacturer's protocol. Absorbance was measured at $450 \mathrm{~nm}$. Data are presented as the mean \pm standard deviation of three separate experiments. For demethylation detection, KLE cells $\left(3 \times 10^{3} /\right.$ well) were plated into 96 -well plates and incubated with complete medium or medium containing $5 \mu \mathrm{mol} 5$-Aza-dC for 1-7 days, then cells underwent CCK-8 assay according to the aforementioned protocol.

Tumorigenesis in vivo. For in vivo experiments, $1 \times 10^{7}$ pBABE-WIF1-KLE and control KLE cells were injected subcutaneously into 12 athymic nude female mice (n=6/group; age, 4 weeks; weight, 18-20 g). The mice were supplied by Beijing Vital River Laboratory Animal Technology Co., Ltd. (Beijing, China). Animals were housed in the animal facility of the Medical College of Shandong University (Shandong, China), at $26-28^{\circ} \mathrm{C}, 40-60 \%$ humidity and a $12 / 12 \mathrm{~h}$ light/dark cycle. Food and water, supplied by Beijing Keao Xieli Feed Co., Ltd. (Beijing, China), were autoclaved prior to feeding and were freely available. Tumor growth was monitored by measurement of the tumor diameter every 4 days from days 14 to 34 following injection. For each time point, the mean tumor volume was calculated for each group of 6 mice. After 34 days, all 12 mice were sacrificed by neck dislocation, tumors were collected and pictures were acquired. All animal experiments were performed with the approval of the Animal Care and Use Committee of Shandong University (Shandong, China).

Statistical analysis. Statistical analysis was performed using SPSS software (version 20.0; IBM Corp., Armonk, NY, USA). Differences in the frequencies of WIF1 methylation or expression between groups were examined using the Pearson's $\chi^{2}$ and Fisher exact probability tests. The survival curves were estimated using the Kaplan-Meier estimate, and the difference in their distribution was evaluated by a log-rank test. Disease-specific survival time was determined by comparing the diagnosis date with the date of mortality caused by EAC. Comparisons in cell density, and the relative levels of mRNA expression, protein expression and tumor size between the two different transfection groups were presented as the mean \pm standard deviation from at least three independent experiments, and were analyzed using a unpaired Student's t-test. $\mathrm{P}<0.05$ was considered to indicate a statistically significant difference.

\section{Results}

Pyrosequencing, methylation and mRNA expression of WIF1 in primary $E A C$. The methylation and mRNA expression status of WIF1 in EAC and control endometrial tissues was determined (Fig. 1). Two out of three EAC cases exhibited significantly high methylation in five $\mathrm{CpG}$ sites (Fig. 1A). WIF1 methylation was observed in 46 of the 106 cases (43.4\%) of EAC tumor tissue by MSP (Table I); however, methylation was only observed in 4 out of $50(8 \%)$ normal endometrial tissue samples; this difference in methylation was statistically significant $(\mathrm{P}<0.05$; Fig. 1B). WIF1 mRNA was detectable in $47.2 \%(50 / 106)$ of neoplastic EAC tissues, which was significantly lower than that observed in non-neoplastic tissues [41/50 (82\%); Fig. 1C]. The WIF1 methylation positivity was inversely related to WIF1 expression positivity $(\mathrm{P}<0.01$; Fig. 1E).

Prognostic value of WIF1 expression and methylation. The protein expression levels of WIF1 in primary EAC and non-tumorous endometrial tissues are presented in Fig. 2A and B. WIF1 expression status was defined as positive or negative based on WIF1 protein level, in order to provide a prognostic value for the disease. Patients bearing tumors with WIF1 methylation or negative WIF1 expression had a shorter overall survival following surgical resection of primary tumors (Figs. 1D and 2C). The 5-year overall survival rates were $53.9 \%$ for patients with tumors exhibiting negative expression of WIF1, as compared with $77.9 \%$ for those with positive WIF1 expression (Fig. 2C). 
Table I. Clinical, pathological and molecular characteristics of endometrial adenocarcinoma cases.

\begin{tabular}{|c|c|c|c|}
\hline Characteristic & $\begin{array}{c}\text { Total EAC } \\
\text { cases } n=106\end{array}$ & $\begin{array}{l}\text { WIF1 methylated } \\
\text { cases } n=46\end{array}$ & $\begin{array}{l}\text { WIF1 unmethylated } \\
\text { cases } n=60\end{array}$ \\
\hline Age, years (mean \pm standard deviation) & $59.2 \pm 10.9$ & $59.7 \pm 10.3$ & $58.1 \pm 11.5$ \\
\hline \multicolumn{4}{|l|}{ Grade of cancer (n) } \\
\hline 1 & $53(50.0 \%)$ & 21 & 32 \\
\hline 2 & $35(33.0 \%)$ & 16 & 19 \\
\hline 3 & $18(17.0 \%)$ & 9 & 9 \\
\hline \multicolumn{4}{|l|}{ FIGO stage (n) } \\
\hline I & $40(37.7 \%)$ & 13 & 27 \\
\hline II & $13(12.3 \%)$ & 6 & 7 \\
\hline III & $37(34.9 \%)$ & 19 & 18 \\
\hline IV & $16(15.1 \%)$ & 8 & 8 \\
\hline \multicolumn{4}{|l|}{ Adjuvant therapy (n) } \\
\hline No & $46(43.4 \%)$ & 17 & 29 \\
\hline Yes & $60(56.7 \%)$ & 29 & 31 \\
\hline
\end{tabular}

Characteristics recorded in patients with EAC $(n=106)$. EAC, endometrial adenocarcinoma; FIGO, Federation of Gynecology and Obstetrics staging; WIF1, Wnt inhibitory factor 1.

A
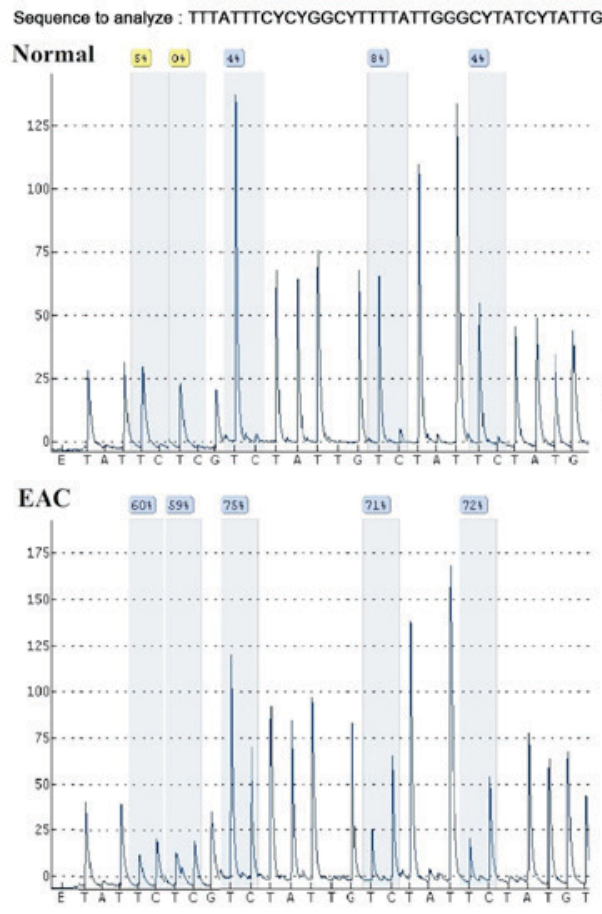

B

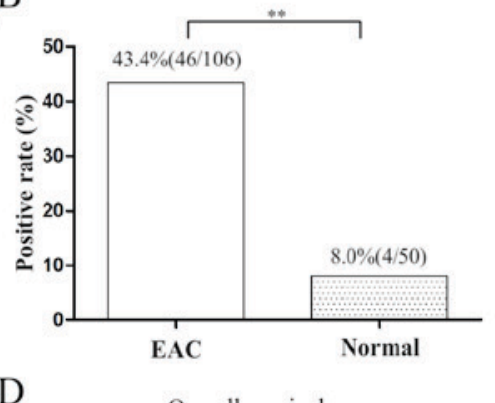

$\mathrm{D}$

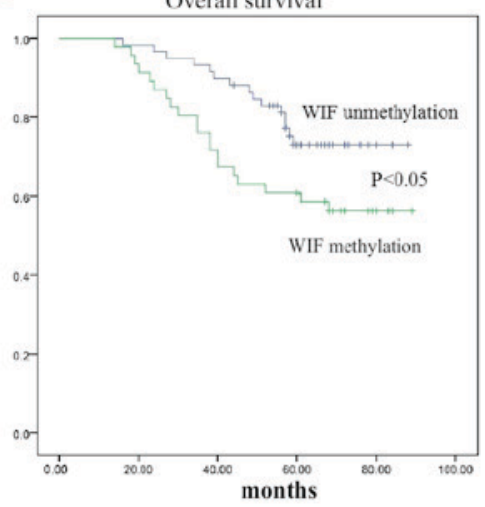

$\mathrm{C}$

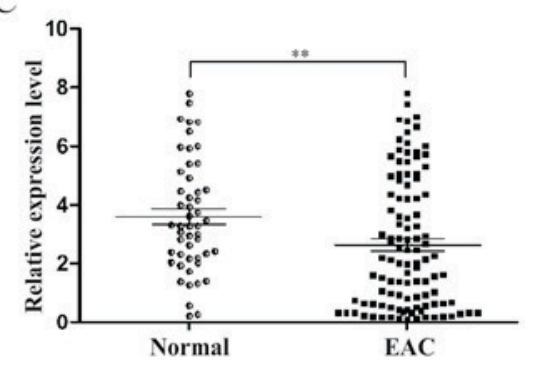

E

\begin{tabular}{|c|c|c|}
\hline Cases & \multicolumn{2}{|c|}{ WIF-1 methylation } \\
\hline & Methylated & Unmethylated \\
\hline WIF-1 expression & & \\
\hline Positive & 12 & 38 \\
\hline Negative & 34 & 22 \\
\hline Kappa & \multicolumn{2}{|c|}{$\mathbf{- 0 . 3 6 9 ( p < 0 . 0 1 )}$} \\
\hline
\end{tabular}

Figure 1. Methylation and mRNA expression status in EAC and control endometrial tissues. (A) Representative pyrosequencing data exhibiting the peaks produced by methylated $\mathrm{CpG}$ sites in normal and EAC samples. (B) Positive methylation rates in patients with EAC and normal controls. (C) Dot plot analysis of mRNA expression. (D) Kaplan-Meier survival analysis for WIF1 methylation in EAC. (E) Associations between methylation and mRNA expression. Data are presented as the mean \pm standard error. ${ }^{* *} \mathrm{P}<0.01$, as indicated. EAC, endometrial adenocarcinoma; WIF1, Wnt inhibitory factor 1 .

Demethylation of the WIF1 promoter restores its expression in the EAC cell line KLE and inhibits cell proliferation. The methylation and expression of WIF1 were detected in KLE cells with or without 5-Aza-dC treatment, in order to identify whether WIF1 silencing is associated with promoter hypermethylation (Fig. 3). KLE cells were treated with 5-Aza-dC, which resulted in a decrease in methylation and the restorationof WIF1 expression (Fig. 3A and C). WNT1, which is the downstream factor of WIF1, was inhibited by restoration of WNT1 (Fig. 3C). In addition, cell proliferation was significantly reduced following 5-Aza-dC treatment in KLE cells (Fig. 3B). 


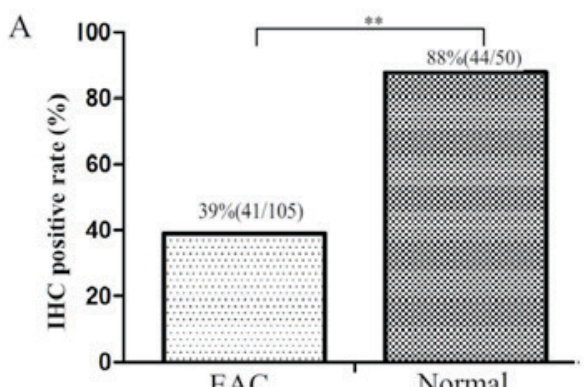

C

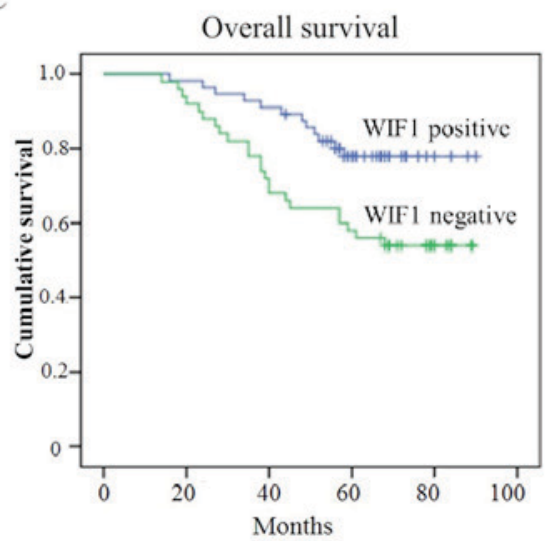

B
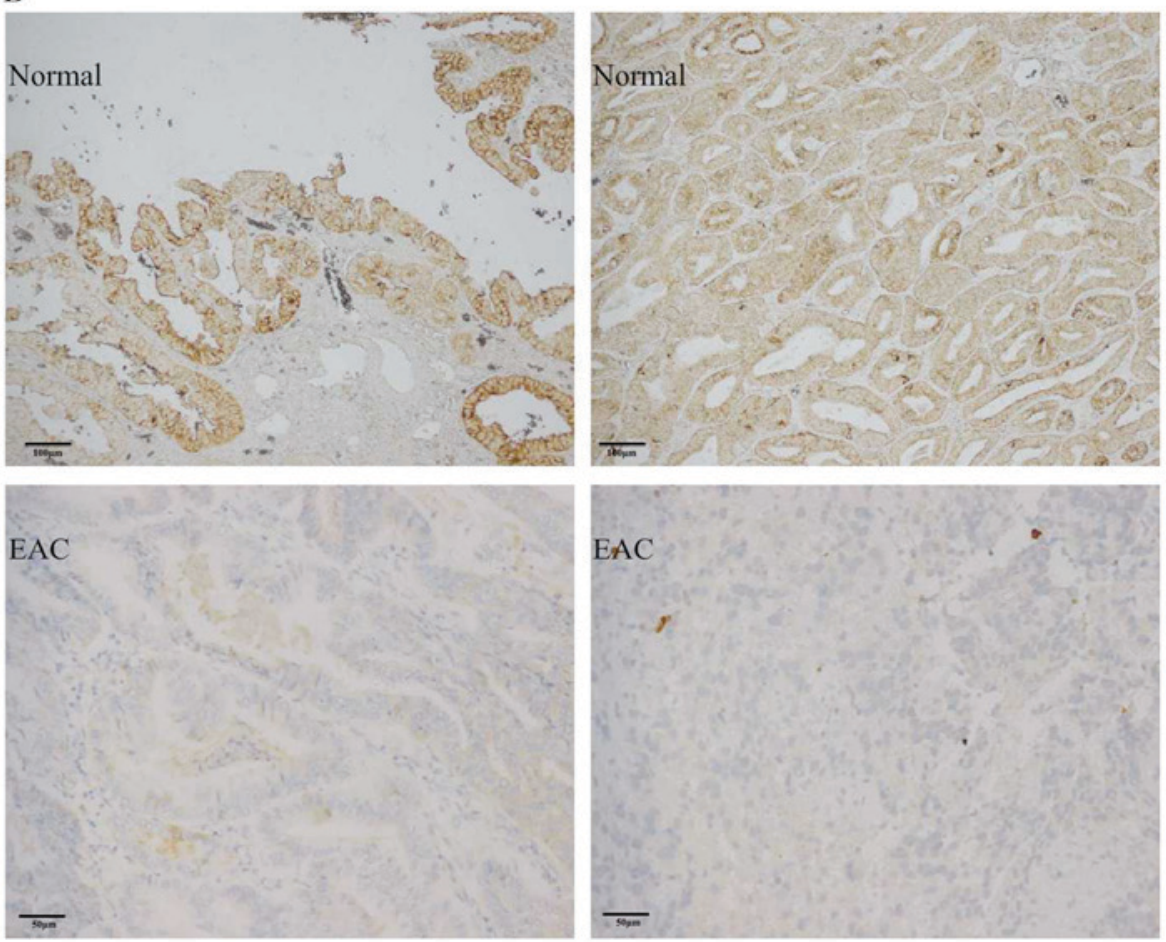

Figure 2.WIF1 protein expression. (A) WIF1 protein expression in EAC and control endometrial tissues as indicated by IHC positive staining. (B) Representative photomicrographs illustrating the IHC staining pattern of WIF1. (C) Kaplan-Meier survival analysis for WIF1 expression in EAC. ${ }^{* *} \mathrm{P}<0.01$, as indicated. EAC, endometrial adenocarcinoma; IHC, immunohistochemistry; WIF1, Wnt inhibitory factor 1.

A

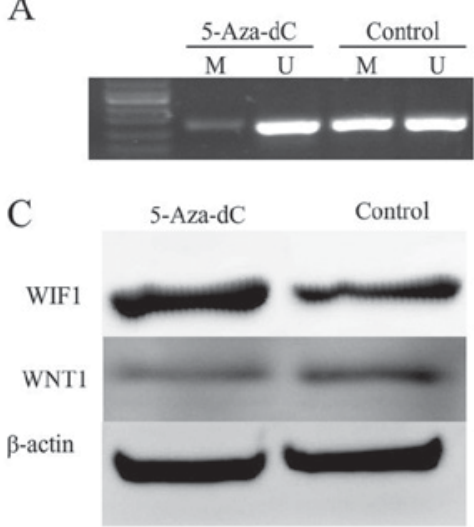

B

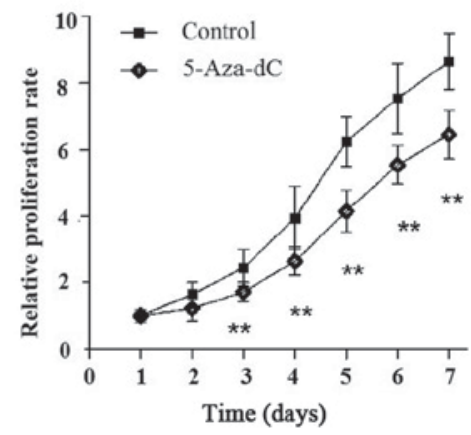

Figure 3. Treatment of the KLE cell line with $30 \mu \mathrm{mol} / 1$ 5-Aza-dC. (A) Methylation rate was detected by methylation-specific polymerase chain reaction following treatment with or without 5-Aza-dC. (B) KLE cell proliferation was examined using a Cell Counting Kit-8 assay. (C) WIF1 and WNT1 expression were detected by western blotting. ${ }^{*} \mathrm{P}<0.01$, as indicated. Data are presented as the mean \pm standard error of mean. 5-Aza-dC, 5-aza-20-deoxycytidine; M, methylated; U, unmethylated; WIF1, Wnt inhibitory factor 1; WNT1, Wnt family member 1 .

WIF1 reduces cell proliferation by reducing $c$-Myc and phosphorylated (p)-ERK in vitro. To examine the effects of restored WIF1 on KLE cell proliferation, cells were stably transfected with pBABE-WIF1 or empty vector. CCK-8 assay revealed that the proliferation of KLE cells transfected with pBABE-WIF1 was significantly inhibited compared with the cells transfected with the control $(\mathrm{P}<0.05$; Fig. 4A). When compared with the control group, western blotting demonstrated that WIF1 overexpression in KLE cells led to downregulation of c-Myc and p-ERK (Fig. 4B). Collectively, these results indicated that WIF1-induced suppression of proliferation in KLE cells may be associated with the inhibition of the transcriptional factor c-Myc and the phosphorylation of ERK.

WIF1 inhibits tumor growth of xenografts. To examine whether WIF1 overexpression could suppress KLE cell growth in vivo, KLE cells transfected with pbabe-puro-WIF1 or pbabe-puro empty vector were subcutaneously inoculated into nude mice ( $n=6 /$ group). The results demonstrated that the size of tumors derived from pBABE-WIF1-overexpressing cells was significantly smaller than those in the control group $(\mathrm{P}<0.05$; Fig. $4 \mathrm{C}$ and $\mathrm{D})$. 
A

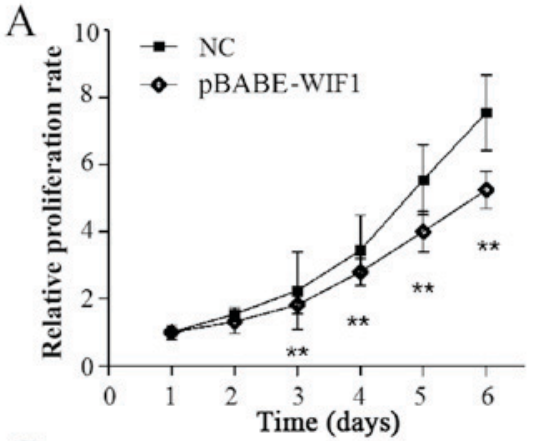

C

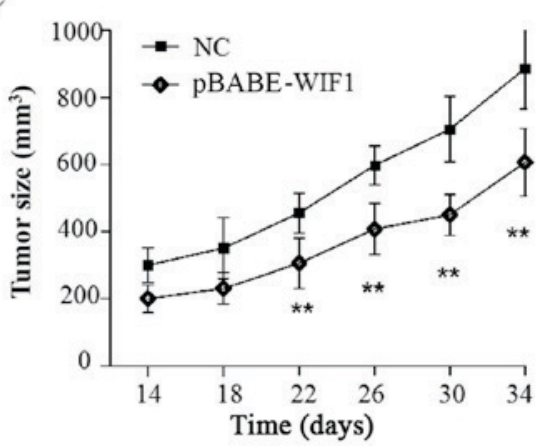

B

WIF1

p-ERK

ERK

c-Myc

$\beta$-actin

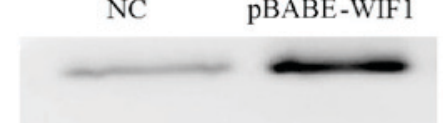

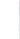
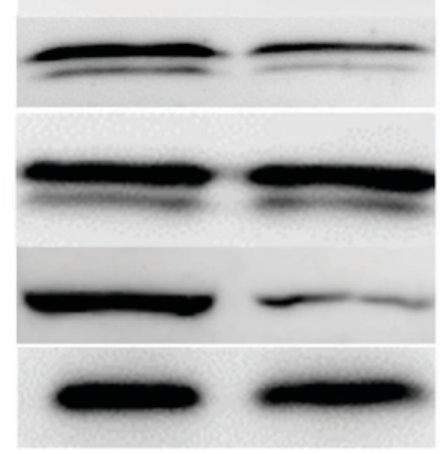

D

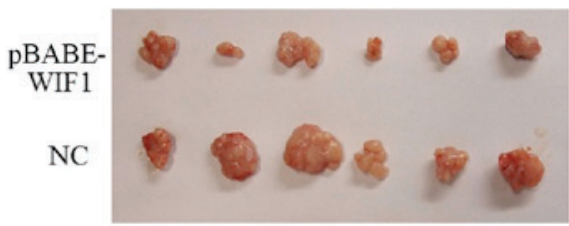

Figure 4. Stable WIF1 overexpression suppresses cell proliferation in vitro and in vitro. (A) Proliferation of pBABE-WIF1-KLE-transfected and control cells was examined using a Cell Counting Kit-8 assay. (B) Western blotting was performed to detect the expression of markers involved in the Wnt signaling pathway. (C) Size of tumors generated in vitro by pBABE-WIF1-KLE and control cells over 34 days. (D) Representative image of tumors generated by pBABE-WIF1-KLE and control cells in vitro. Tumors were resected after 34 days. ${ }^{* *} \mathrm{P}<0.01$, as indicated. Data are presented as the mean \pm standard error of mean. ERK, extracellular signal-regulated kinase; NC, pbabe empty; p-, phosphorylated; WIF1, Wnt inhibitory factor 1.

\section{Discussion}

The Wnt signaling pathway, which is evolutionarily well conserved, has a crucial role in embryonic development and in numerous cancer-associated processes $(11,12)$. Alterations in the Wnt $/ \beta$-catenin signaling pathway have been identified in $\leq 50 \%$ of EAC cases (13). Nuclear accumulation of $\beta$-catenin, which is an activated factor of $\mathrm{Wnt} / \beta$-catenin signaling, has been detected in $\leq 47 \%$ of EAC cases (13-15). The gain-of-function mutation present in the $\beta$-catenin gene appears to be an important mechanism by which Wnt/ $\beta$-catenin signaling is activated, and has been observed in $\sim 25 \%$ of EAC cases $(9,15,16)$.

WIF1 is a secreted Wnt antagonist, which can directly bind to Wnts in order to prevent signaling $(17,18)$. WIF1 is often downregulated by promoter hypermethylation in numerous types of human cancer, including prostate (19), cervix (20), esophagus (21) and others $(22,23)$. The present study demonstrated that WIF1 downregulation is a widespread event in human EAC oncogenesis, which occurs frequently due to promoter hypermethylation. This is consistent with previous studies in other cancer types (19-23), which reported that promoter methylation is the major mechanism for the inactivation of this tumor suppressor gene (24). The frequency of WIF1 methylation in primary EAC identified in the present study was $43.4 \%$. To further explore the role of DNA methylation in the transcriptional repression of the WIF1 gene, KLE cells were treated with 5 -Aza-dC. The results demonstrated that 5-Aza-dC was able to restore the expression of WIF1 in the KLE cell line and inhibit cell proliferation.
In the present study, WIF1 protein expression was associated with good survival rates in EAC. This is similar to a previous study in hepatocellular carcinoma (HCC), which reported that WIF1 protein expression, but not methylation of WIF1, is a predictor of good patient outcomes in those undergoing resection of HCC (25). In addition, WIF1 methylation was reported to act as a strong prognostic factor in overall survival, and the increased methylation index was significantly associated with decreased relapse-free survival and overall survival of non-small cell lung cancer (26). However, increased WIF1 promoter methylation and decreased WIF1 protein expression were not associated with human astrocytoma patient survival (27).

By transfecting cell lines with a WIF1-expressing plasmid, the present study has demonstrated that ectopic expression of WIF1 in EAC cell lines may inhibit endometrial cancer cell proliferation. This inhibition of cell proliferation is consistent with the inhibition of cell growth by WIF1 as previously shown in other cancer cell lines, indicating that WIF1 acts as a functional tumor suppressor $(6,28)$. The present study also provided evidence to suggest that WIF1 exerts its tumor suppressor functions by downregulating the intracellular protein levels of c-Myc and p-ERK, which are important $\mathrm{Wnt} / \beta$-catenin transcriptional target genes.

In conclusion, to the best of our knowledge, the present study is the first to report that the WIF1 gene is frequently hypermethylated in EAC, which is an important mechanism to silence WIF1 gene expression. Conversely, restoration of WIF1 expression in EAC cells was able to inhibit cell growth in vitro and in vivo. These results suggested that WIF1 may be 
frequently inactivated by promoter methylation and therefore, may be considered a candidate tumor suppressor in EAC.

\section{Acknowledgements}

The present study was supported by the Youth Fund of the Second Hospital of Shandong University (grant no. 26010275618056).

\section{References}

1. Siegel RL, Miller KD and Jemal A: Cancer statistics, 2015. CA Cancer J Clin 65: 5-29, 2015.

2. Murali R, Soslow RA and Weigelt B: Classification of endometrial carcinoma: More than two types. Lancet Oncol 15: e268-e278, 2014

3. Bokhman JV: Two pathogenetic types of endometrial carcinoma. Gynecol Oncol 15: 10-17, 1983.

4. MacDonald BT, Tamai K and He X: Wnt/beta-catenin signaling: Components, mechanisms, and diseases. Dev Cell 17: 9-26, 2009.

5. Polakis P: Wnt signaling in cancer. Cold Spring Harbor perspectives in biology 4: pii:a008052, 2012.

6. Taniguchi $\mathrm{H}$, Yamamoto $\mathrm{H}$, Hirata $\mathrm{T}$, Miyamoto $\mathrm{N}$, Oki M, Nosho K, Adachi Y, Endo T, Imai KA and Shinomura Y: Frequent epigenetic inactivation of Wnt inhibitory factor-1 in human gastrointestinal cancers. Oncogene 24: 7946-7952, 2005.

7. Yoshino M, Suzuki M, Tian L, Moriya Y, Hoshino H, Okamoto T, Yoshida S, Shibuya K and Yoshino I: Promoter hypermethylation of the p16 and Wif-1 genes as an independent prognostic marker in stage IA non-small cell lung cancers. Int J Oncol 35 : 1201-1209, 2009

8. Urakami S, Shiina H, Enokida $H$, Kawakami T, Tokizane $T$, Ogishima T, Tanaka Y, Li LC, Ribeiro-Filho LA, Terashima M, et al: Epigenetic inactivation of Wnt inhibitory factor-1 plays an important role in bladder cancer through aberrant canonical Wnt/beta-catenin signaling pathway. Clin Cancer Res 12: 383-391, 2006.

9. Liu Y, Patel L, Mills GB, Lu KH, Sood AK, Ding L, Kucherlapati R, Mardis ER, Levine DA, Shmulevich I, et al: Clinical significance of CTNNB1 mutation and Wnt pathway activation in endometrioid endometrial carcinoma. J Natl Cancer Inst 106: pii:dju245, 2014.

10. Livak KJ and Schmittgen TD: Analysis of relative gene expression data using real-time quantitative PCR and the 2(-Delta Delta $\mathrm{C}(\mathrm{T})$ ) method. Methods 25: 402-408, 2001.

11. Huelsken J, Vogel R, Erdmann B, Cotsarelis G and Birchmeier W: Beta-catenin controls hair follicle morphogenesis and stem cell differentiation in the skin. Cell 105: 533-545, 2001.

12. Weigelt $B$ and Banerjee S: Molecular targets and targeted therapeutics in endometrial cancer. Curr Opin Oncol 24: 554-563, 2012.

13. Klaus A and Birchmeier W: Wnt signalling and its impact on development and cancer. Nat Rev Cancer 8: 387-398, 2008.

14. Dedes KJ, Wetterskog D, Ashworth A, Kaye SB and Reis-Filho JS: Emerging therapeutic targets in endometrial cancer. Nat Rev Clin Oncol 8: 261-271, 2011.
15. Matias-Guiu X and Prat J: Molecular pathology of endometrial carcinoma. Histopathology 62: 111-123, 2013.

16. McConechy MK, Ding J, Cheang MC, Wiegand K, Senz J, Tone A, Yang W, Prentice L, Tse K, Zeng T, et al: Use of mutation profiles to refine the classification of endometrial carcinomas. J Pathol 228: 20-30, 2012.

17. Kawano Y and Kypta R: Secreted antagonists of the Wnt signalling pathway. J Cell Sci 116: 2627-2634, 2003.

18. Tamai K, Semenov M, Kato Y, Spokony R, Liu C, Katsuyama Y, Hess F, Saint-Jeannet JP and He X: LDL-receptor-related proteins in Wnt signal transduction. Nature 407: 530-535, 2000.

19. Yee DS, Tang Y, Li X, Liu Z, Guo Y, Ghaffar S, McQueen P, Atreya D, Xie J, Simoneau AR, et al: The Wnt inhibitory factor 1 restoration in prostate cancer cells was associated with reduced tumor growth, decreased capacity of cell migration and invasion and a reversal of epithelial to mesenchymal transition. Mol Cancer 9: 162, 2010.

20. Ramachandran I, Thavathiru E, Ramalingam S, Natarajan G, Mills WK, Benbrook DM, Zuna R, Lightfoot S, Reis A, Anant S and Queimado L: Wnt inhibitory factor 1 induces apoptosis and inhibits cervical cancer growth, invasion and angiogenesis in vivo. Oncogene 31: 2725-2737, 2012.

21. Yang SH, Li SL, Dong ZM and Kan QC: Epigenetic inactivation of Wnt inhibitory factor-1 in human esophageal squamous cell carcinoma. Oncol Res 20: 123-130, 2012.

22. Kawakami K, Hirata H, Yamamura S, Kikuno N, Saini S, Majid S, Tanaka Y, Kawamoto K, Enokida H, Nakagawa M and Dahiya R: Functional significance of Wnt inhibitory factor-1 gene in kidney cancer. Cancer Res 69: 8603-8610, 2009.

23. Kansara M, Tsang M, Kodjabachian L, Sims NA, Trivett MK, Ehrich M, Dobrovic A, Slavin J, Choong PF, Simmons PJ, et al: Wnt inhibitory factor 1 is epigenetically silenced in human osteosarcoma, and targeted disruption accelerates osteosarcomagenesis in mice. J Clin Invest 119: 837-851, 2009.

24. Malinauskas T, Aricescu AR, Lu W, Siebold C and Jones EY: Modular mechanism of Wnt signaling inhibition by Wnt inhibitory factor 1. Nat Struct Mol Biol 18: 886-893, 2011.

25. Huang L, Li MX, Wang L, Li BK, Chen GH, He LR, Xu L and Yuan YF: Prognostic value of Wnt inhibitory factor-1 expression in hepatocellular carcinoma that is independent of gene methylation. Tumour Biol 32: 233-240, 2011.

26. Yoshino M, Suzuki M, Tian L, Moriya Y, Hoshino H, Okamoto T, Yoshida S, Shibuya K and Yoshino I: Promoter hypermethylation of the p16 and Wif-1 genes as an independent prognostic marker in stage IA non-small cell lung cancers. Int J Oncol 35: 1201-1209, 2009

27. Kim SA, Kwak J, Nam HY, Chun SM, Lee BW, Lee HJ, Khang SK and Kim SW: Promoter methylation of WNT inhibitory factor- 1 and expression pattern of $\mathrm{WNT} / \beta$-catenin pathway in human astrocytoma: Pathologic and prognostic correlations. Mod Pathol 26: 626-639, 2013.

28. Zhang J, Zhou B, Liu Y, Chen K, Bao P, Wang Y, Wang J, Zhou Z, Sun X and Li Y: Wnt inhibitory factor-1 functions as a tumor suppressor through modulating $\mathrm{Wnt} / \beta$-catenin signaling in neuroblastoma. Cancer Lett 348: 12-19, 2014. 